\title{
BRITISH MEDICAL JOURNAL
}

\author{
LONDON SATURDAY NOVEMBER 11941
}

\section{THE CORRELATION OF PATHOLOGY, PHYSiCAl SIGNS, AND X-RAY APPEAR- ANCES IN THE DEVELOPMENT OF LUNG CAVITATION*}

BY

\author{
R. R. TRAIL, M.C., M.A., M.D., F.R.C.P. \\ Physician, Royal Chest Hospital, London; Physician to Surgical Unit, Papworth; Wing Commander, R.A.F.V.R.
}

The correlation of pathological findings with physical and radiological signs is an unfailing source of interest to the diagnostician. In diseases of the chest such a study is a fascinating game. It is still a game, in the nature of a jigsaw puzzle, in that the answer we continue to seek assiduously in most of the problems set is the individual living pathology, the pathogenesis at the moment of diagnosis, which shall act as the basis of that wider problem of individual treatment, ever the ultimate aim and true reward of the happy physician. Not every player plays the game with the same pieces. Some claim ability to find the answer with a limited number; some even despise certain sets of pieces.

Controversial articles on the value of mass radiography recently appearing in the medical press serve to illustrate this point. Certain writers have professed to be independent of $x$-ray aids in the diagnosis of tuberculosis; they say that to them clinical history and stethoscopic signs are all-sufficient. Some have gone to the other extreme and have relegated the stethoscope to the scrap-heap. Both classes may, as individuals, be entirely truthful in their statements, the first having ears to hear and the second eyes to see. We do well, however, to ignore deaf advocates of perpetual ear stops, however well they can see, and preachers of permanent blinkers, however well they can hear. The "useless" club in our golf outfit is too often the one we have never learnt to use. The truth is, we still require every possible aid-pathological, radiological, and stethoscopic - in the diagnosis of chest disease if we would belong to the class of the happy physician "who stops, and who looks, and who listens."

Let us consider how these principles affect the discovery of cavitation, throughout its development, in bronchiectasis, abscess of lung, and tuberculosis.

\section{Bronchiectasis}

We will leave aside the much-discussed subject of congenital bronchiectasis and confine ourselves to the disease in its acquired form. Even here there is controversy on the intrabronchial or extrabronchial origin, but it is generally accepted that the first recognizable pathological change is dilatation of the bronchus, due to obstruction plus infection, and that this dilatation is the first step in the production of the bronchiectatic abscess cavity.

In children the process is usually very acute, taking the form of ulcerative bronchitis, which breaks down the bronchial wall and invades the lung substance so rapidly that all the clinical and radiological findings of an advanced stage of adolescent and adult forms of the disease present almost from the first symptomatic evidences.

* Read before the Ely Medical Society of the R.A.F. on June 8,1941 .
From adolescence onwards the progress of the disease is generally much slower. Here chronic upper respiratory tract infections and chronic bronchitis are early secondary invaders of the damaged and weakened bronchi, and cause a loss of muscle tone that in its turn produces further dilatation. The normal action of respiratory widening by comparative negative intrathoracic pressure, exerted by the pleural vacuum, is not now followed by normal contraction of undamaged elastic tissue, and this vicious circle is continued by the resultant accumulation of debris in the lumen, interfering by its dead weight with the natural sweeping movements of the cilia.

Microscopical examination shows affected bronchi to be chronically inflamed and slowly breaking down and atrophying. In some parts only the epithelium will be affected ; in others muscle and elastic tissue are fast disappearing ; while in an advanced area every element of the bronchial wall is mixed beyond recognition into a dense fibrous mass, and the ciliated columnar epithelium is beginning to show squamous-cell infiltration. Surrounding areas of the lung show compensatory emphysema.

At this stage the physical and $x$-ray findings are in most cases bilateral and basal. The heart is central; there is no mediastinal displacement; and clubbing of the fingers, if recognizably present, is still at the first stage. This consists of an excess of tissue at the root of the nails-cyanotic, and with an unnatural shine as from continual polishing. The movement of the chest is heaving in the lower zones rather than lateral, owing to compensatory emphysema. The note to light percussion is impaired, but the overlying emphysema may very easily produce a deceptive tympany. In most cases, as there is still some airway in the bronchi, rhonchi and sibili are heard through the whole inspiratory phase ; but they are most insistent in the first half of the phase, and return equally loudly in the middle and last phases of expiration. If such added sounds remain after cough they are lessened in intensity and practically confined to the early phase of inspiration. In addition, there are heard throughout the whole of inspiration, both on ordinary breathing and after cough, fine to coarse sounds of a dry character. The characteristic appearance of increased striation on the film and the microscopical finding of increased deposition of connective tissue in and around the bronchi and their associated alveoli have taught us to recognize these as the stethoscopic finding of established fibrosis.

The $x$-ray film taken at this stage shows heavy, rather fluffy linear shadows towards both bases, irregular in their loss of translucency, but more or less faithful to the normal branchings of the larger bronchial bundles. We are viewing the increased blood supply of chronically. inflamed bronchi, and occasionally slight invasions of lung tissue through penetrated bronchial walls. 
The introduction of lipiodol would show enlarged bronchi, slowly increasing in width from the upper border of the affected area downwards towards the bases, occasionally pulled into small nodular sacculations, and now and again without true definition in the shadows of their edges.

The further stages, so well demonstrated in children, constitute a picture of rapid deterioration. Increased inflammation produces complete destruction of the elastic and muscle fibres; resultant debris brings occlusion of the lumen; the cilia give up the unequal struggle, and their consequent paresis is followed by organization, shown in the replacement of their columnar by squamous-cell epithelium. Endarteritis of the vessels in the walls brings increased fibrosis ; areas of atelectasis in the alveoli of the neighbouring bronchioles add to the negative intrathoracic pressure already pulling the dilated lumen into saccules, until finally there is complete destruction of all elements, including the cartilage, and perforation brings further sepsis to surrounding parenchyma.

The $x$-ray picture changes with these developments. Linear densities previously noted become more irregular and extensive, and fibrosis is demonstrated by the shift of the heart and mediastinum to the affected or more affected side. Septic extensions through the bronchi to lung tissue are seen as ill-defined areas of loss of translucency, like blobs of cotton-wool, around and intimately mixed with the shadows of chronically inflamed and dilated bronchi. These, at first small and more or less discrete in the acute state, later become confluent, and are often entirely similar to the shadow now called that of pneumonitis ; they have, however, a distinctive and diagnostic difference in that the accompanying fibrosis produces mediastinal shift. Such shadows come and go with the onset of and recovery from the well-known exacerbations of the established disease. But temporary recovery only adds to the vicious circle of dilatation of the bronchi as new areas of atelectasis repeat the previous process, so that the basic and diagnostic appearances of marked peribronchial vascularity are still heavier, more grossly contorted, and less and less associated with the linear distribution of normal bronchi.

Examination with lipiodol will show exaggerations of the anomalies previously noted on its introduction. Enlargement is much more pronounced, the edges of the bronchi will be more confused and nodular; some will show nothing comparable with normal outlines, but be replaced by gross sacculations, while still others will be completely indistinguishable and lost in a ragged cavity invading surrounding parenchyma to greater or lesser degree.

Physical signs have also altered. The mediastinum has moved to the affected or more affected side. Such shift is most easily demonstrated by the "sterno-mastoid sign." This consists in gentle palpation of the band of the sternomastoid towards its origin at the inner end of the clavicle while the patient is lying or sitting in a relaxed position with the head central to the thorax. On that side to which the mediastinum is either pulled or pushed there will be found marked tension. The scaleni on the same side of the neck may be similarly affected. Simple scoliosis can produce such a sign in minor degree, but can be excluded very easily by chest inspection alone.

Developed osteo-arthropathy is now an evident sign. This is "septic" in type: the whole terminal phalanx is involved, enlarged in all diameters, gross, clumsy, and "drumstick" in appearance. Such clubbing is entirely different from the usual finding in tuberculosis, where the enlargement is "dorsal-palmar" in type, and tends to give a more claw-like, tapered, and refined look to the fingers.
Marked lack of movement and dullness are present over the affected areas. Added sounds take on a new and distinctive character. Coarse rhonchi may be heard between exacerbations, as there are areas of incomplete blockage of the lumen ; but the most diagnostic signs in such conditions are rales, exceedingly coarse and metallic, best heard in the first phase of inspiration, and remaining post-tussive. They tail off in the second half of inspiration to those already described as evidence of fibrosis, and tell us our patient has reached the stage of sacculation and abscess-cavity formation. Destruction of all elements of the bronchial wall is active and progressive.

During exacerbations the areas with surrounding pneumonitis may be quite silent owing to complete blockage of the lumen and the filling of communicating lobules with pneumonic exudate, but examination repeated after a period of rest in bed will show further increase in numbers and intensity of the characteristic metallic rales, which encroach more and more into the middle phase of inspiration, showing that the patient has now developed lung abscess of bronchiectatic origin.

\section{Lung Abscess}

The cavitation of lung abscess which is not bronchiectatic in origin is entirely different in physical and radiological signs throughout its development, although its pathology is rather similar in its main features. We have seen that in bronchiectatic cavity formation the primary process may be followed from an intrabronchial origin and that the abscess is an involvement of parenchyma by extension. Such an abscess cavity is met with in overflow, by proximity plus pressure, in cases of empyema, and is common in the interlobar type. It is similarly found after aspiration of foreign bodies and septic material into the bronchi-for example, after operations on the upper respiratory tract.

We will not deal here with multiple small abscess formation due to dissemination of septic emboli through the blood stream, where the breaking down is an extension of connective-tissue destruction, but will confine ourselves to that type of abscess which has its beginnings in the alveolar spaces and follows bronchopneumonia and, more rarely, pneumonia as the primary disease. We find that the branches of the more vertical right lower bronchi are more frequently involved than the left in bronchopneumonic cases: for the same reason the upper lobes are more rarely affected than the lower. The site of the lesion is far more often peripheral than central. The majority of the abscesses are superficial, and so immediately under the pleura that it is affected from the onset. At an early stage they are not easily determined in outline post mortem, being merely mushy gangrenous-looking areas in the middle of bunches of alveoli occluded by typical pneumonic exudate. There is, however, a very distinct change in the film associated with the causative disease. If this has been pneumonia, the loss of translucency, previously equal from periphery to heart shadow at any one level throughout its extent, becomes greater in the abscess area, and comparatively less, to entire absence, in other areas of the affected lobe. This denser area is generally in the outer zone of the lung field. There is no shift of the trachea or mediastinum to the diseased side.

If the previous condition has been bronchopneumonia the alteration in shadow is just as dramatic. In place of scattered areas of loss of translucency, never confluent and yet never entirely separated by normal translucent areas, and generally much more evident over one lower zone than the other, there appears the shadow described above. This is confluent, ill defined in its edges, and heaviest in its central zone. 
To physical examination there is definite lack of movement of the affected side and a fairly limited impairment of note. The diagnostic stethoscopic sign is a coarse pleural rub, usually far more extensive than the area of dullness, and out of all proportion to symptoms ; indeed, it may be entirely painless. Such a finding over the right upper lobe, especially if it be post-operative to upper sinus infection and anterior and below the clavicle, is almost by itself diagnostic of abscess formation. When actual cavitation occurs the pleural rub either becomes very localized and annular in distribution to the underlying lung lesion, or disappears entirely, as the pleura has become adherent and thickened in its layers. But a new and equally diagnostic stethoscopic sign appears.

In pneumonic cases, in place of the redux crepitations of reopening alveoli, coarse metallic rales of the character already described in bronchiectatic abscess are heard, this time most insistent in the mid-phase of inspiration. They are, so far as their appearance in inspiration is concerned, the signs we heard in the last stages of abscess formation in bronchiectasis, where the lung involvement was secondary to bronchial sacculation: there they were a continuation to the mid-phase from the first phase of inspiration; now they begin in the mid-phase as lung involvement by sepsis precedes bronchial involvement. Accompanying them throughout inspiration there may be high-pitched sibili and coarse rhonchi, of the type commonly found in partial blockage of the bronchi. Where bronchopneumonia has been the preceding disease these metallic rales replace the moist rales, of definitely less intensity, heard in the same phase of inspiration in all ordinary uncomplicated cases.

There is still at this stage no shift of the mediastinum to either physical or radiological findings, but the latter change within the area of the actual lesion. Towards the centre of the dense area a translucency becomes apparent, either ill defined or with a linear edging only, oval or rounded in shape. A fluid level may appear, but is unusual at this stage. Its outline is no more marked by viewing the film at the distance of some six feet than it is on close inspection, nor does any change appear at differing distances in the surrounding area of loss of translucency. In both such features, as we shall see later, the film differs considerably from that of fibro-caseous tuberculosis with cavitation. The reason for this we can find by examination of the cavity at such a stage post mortem. The wall is not fibrotic but ill defined and ragged, and is heavily infiltrated with large mononuclear phagocytes and polymorphs. The surrounding alveoli are filled with typical pneumonic exudate. Should such a cavity not be evacuated further changes will appear. Generally within three to four weeks of its first formation dense fibrous tissue appears in its wall, shutting it off from surrounding lung. Similarly, organization of their pneumonic exudate is followed by induration of surrounding alveoli. Bronchi become infected and bronchiectasis supervenes ; we are witnessing the reverse process, albeit in a restricted area, which we followed in the formation of abscess cavity in primary bronchiectasis.

Film now shows a well-defined cavity wall, rounder and smoother in outline than the chronic tuberculous cavity, and more apt to contain a fluid level. Further in comparison with such a cavity, it changes more suddenly from its well-defined inner wall to a generalized loss of translucency in surrounding lung tissue, and therefore still alters but little by distant viewing as against close inspection. Pathology tells us why: induration and organization are confined in lung abscess to alveoli close to the circumscribed lesion, but in fibro-caseous tuberculosis acinar collapse and fibrosis are widespread in comparison, and generally scattered throughout at least the major part of the diseased lobe in which the cavity develops.

Commencing mediastinal shift, now evident on the film, can be discovered by physical examination, and is due to the increasing fibrosis, which adds its stethoscopic signs, already described, to those of the still more insistent metallic rales. They are a definite danger signal ; they show that the basis is laid for complicating bronchiectasis, which may persist even with apparent recovery as judged by symptoms. Clubbing of septic type now appears so rapidly that changes can be noted almost from day to day up to gross "drumstick" terminal phalanges.

Disappearance of the annular shadow to ordinary film examination may be entirely misleading in summation of prognosis. Tomography or examination with lipiodol will often show that all that has happened is that the primary lung abscess is replaced by a bronchiectatic abscess, communicating with a dilated and infected bronchus. Herein lies the explanation of what is often misread as a reexacerbation of the primary symptoms, byt is really a secondary and dangerous bronchiectatic process. Stethoscopic signs will aid us to this conclusion. Just as the progress in primary bronchiectasis from dilatation to lung involvement was marked by the forward march of metallic rales from the beginning of inspiration to beyond its middle phase, so is the backward progress now evident, from alveolar spaces to bronchial tube, by the retrogression from mid-phase to beginning of inspiration of these same characteristic sounds.

\section{Tuberculosis}

The progress of tuberculosis differs widely from that of both lung abscess and bronchiectasis in physical signs, radiological findings, and pathology. The key to the understanding of all changes leading up to tuberculous cavitation lies in the study of the behaviour of the acini. Until their caseous content breaks down they are completely silent areas, although they impose a lack of movement on the affected side far beyond their actual extent. But Nature attempts to evacuate them immediately they begin to disintegrate, and each discharges into its corresponding bronchiolus respiratorius. Now appears the earliest stethoscopic sign of active tubercle in a fine sibilus heard in the second half of inspiration. As it is increased in intensity the deeper the inspiration, and reaches its loudest and its highest pitch in the last third of inspiration, it is entirely different from the sounds of ordinary bronchitis.

We have now seen how all these differing sounds, interfering with normal inspiration in differing phases, seem to have a definite correlation with the actual site of the initial lesion in each disease producing them. Bronchial dilatation in early bronchiectasis interferes with the first phase ; lobular lesions in lung abscess with the mid-phase ; and acinar lesions in tuberculosis with the last phase.

A film taken before disintegration of acinar lesions begins is generally of one of two main forms. The first will be typical of the fibro-caseous or proliferative form of the disease, and present a rounded area of loss of translucency; usually in the subclavicular region or near the apex of the lower lobe, on one side of the chest. This is known as the "Assmann focus." The acute, non-fibrous, or exudative type of infiltration will present, on the average, many areas not unlike bronchopneumonia. Although more woolly in their edges, they have more clearly defined translucencies between them than is the case in simple bronchopneumonia. In addition they are more numerous, and are generally more marked in or are confined to the upper and middle zones of the lung fields, as against ordinary bronchopneumonic distribution in the lower lobes 
It is necessary to follow separately these two types of disease in their progress towards established cavitation.

\section{Acute Exudative Tuberculosis}

Acute disease seldom produces a large cavity, although several individual vomicae may appear as if simultaneously, or in quick succession. This is because by its nature it lacks the organization, collapse, and consequent increase in intrathoracic negative pressure characteristic of the proliferative type. The inflammatory exudate filling the acini caseates and disintegrates freely, uncontrolled by fibrous tissue and its accompanying numerous giant cells. No barrier is set round it to protect surrounding lung tissue, and as a result disintegrated tissue fills the bronchi, and bacilli flood the lymph nodes in increasing numbers, causing them in turn to caseate rapidly. The disintegrating area has a moth-eaten, ragged outline, but now and again the process is so acute as to give the impression of a large "ticket-punch" hole. The pleura immediately adjacent is actively inflamed.

On the film such a cavity will appear as an area of comparative translucency among the scattered shadows already described. Sometimes it has no clearly defined edge beyond a ragged outline ; but now and again a definite wall is seen, heavy-looking and of the density of pneumonic exudate, which, we have noted, is indeed its structure. It will then differ quite distinctly from that of chronic lung abscess on the one hand and the chronic tuberculous cavity on the other. It lacks the defined, organized inner edge of chronic abscess and does not appear within a circumscribed area of equal loss of translucency in an otherwise generally uninfected lobe. A later description of the chronic tuberculous cavity will be sufficient aid in this differential diagnosis.

Heavy hilar shadows will be noted, indistinct and confused, although the right tracheo-bronchial group of glands may show up as a separate and fairly well defined entity. As there is no fibrosis, shift of the mediastinum is not present towards either side of the chest.

Physical signs are mainly stethoscopic. Clubbing of the fingers is generally absent beyond its first manifestations. There is little true dullness; lung movement is poor. The preponderant part of the infiltrated area is usually silent, although here and there the diagnostic sign of active infiltration will be heard. Over the site of the vomica localized pleural friction may be heard, but the diagnostic sign of active cavitation consists in a very circumscribed coarse bronchitis, which gives the impression to the listener that it comes from the depths of the lung tissue. $\mathrm{He}$ is hearing a true tuberculous bronchitis by the flooding of the bronchi with caseating exudate.

\section{Chronic Fibro-caseous Tuberculosis}

We have already seen that the usual site of the earliest lesion in proliferative tuberculosis is subapical in the upper lobe. This is the usual site of its cavity, although by the time of its formation infiltration and fibrosis have, in the majority of cases, spread far into the lower lung fields.

Sometimes the Assmann focus breaks almost immediately into cavity formation. It then shows on the film as a welldefined annular shadow, not thick in its outline, and usually with a fluid level. The average case, however, shows a slow disappearance of its regular annular outline: its edge becomes confused, its loss of translucency is no longer uniform, and its lower edges merge into shadows made by the increased blood supply of draining bronchi tracking towards the hilum. Already a slight but definite shift of the mediastinum towards the affected side can be seen, and viewing the films at some distance will throw into relief against a background of generalized loss of translucency bunches of acini, organized or collapsed, and starting to fuse. This is because, from the first, fibrosis has attempted to keep pace with infiltration. Tuberculous granulation tissue has entered the bronchioles; empty acini have collapsed; others are unable to rid themselves of their exudate, which proceeds from caseation to fibrosis. The bronchial wall weakens, dilates, loses its powers of elastic recovery in expiration, and is pulled more and more outwards by the intrathoracic pressure, which is now aided by the surrounding organization. Neighbouring affected acini combine to a solid fused surround of the advancing cavity : more distant ones are scattered throughout the diseased portion of the lung amid more active and less fibrotic areas. Surrounding lung tissue shows much fibrosis. Bronchi show thickened walls, and overlying pleura is adherent, its layers being organized and increased in depth, often to a considerable extent.

Physical signs are in keeping. Typical tuberculous clubbing is present in a large proportion of cases, although in very advanced and long-standing cases superimposed lung infections may make it approximate to the septic type. There is marked displacement of the mediastinum to the affected side, or a less marked but appreciable shift to the more affected side in bilateral disease.

While the signs of active infiltration remain at the advancing edges of active areas, and the signs of fibrosis may be heard throughout the diseased portions of the lungs, these are generally restricted in extent, as against the real amount of involvement found by film examination. A fairly safe rule of thumb appears to be that signs heard above the clavicle mean that at least one-third of the lung is involved, and that signs heard below the clavicle mean that at least half of the lung is involved. Where laryngeal tuberculosis is present we may estimate the true extent as roughly three times that of our stethoscopic findings.

The development of the cavity is signified by the addition to these sounds of coarse metallic rales, again of the same character and quality as those noted in lung abscess and bronchiectasis, but now heard in the last third of inspiration, increasing in intensity the deeper the breath, and remaining, and even increasing in number and coarseness, after cough. In addition, many cases have showers of fine dry-sounding crepitations with the act of coughing, disappearing immediately inspiration begins. They are evidence of thickening and adhering pleura, which is shown on the film as a generalized, even, slight loss of translucency over the infiltrated and fibrotic lung.

The chronic tuberculous cavity shows up on $x$-ray examination as no other cavity does. It has usually $a^{\bullet}$ fairly wide but always well-defined wall, which on close inspection may be hazy in its distal borders, where changing densities show how the scattered nodules of organized and collapsed acini of the barrier are lying in a background of fibrosed lung and covered by thickened pleura. Viewing at a distance, however, throws these densities into strong relief against the background, thus producing a picture quite distinctive, and in itself diagnostic, of fibro-caseous tuberculosis.

\section{Conclusions}

Such, then, is an attempt at correlation of pathology with physical and radiological findings in one small part of the fascinating study of chest disease. It is, as indeed it must be, an individual attempt. To return to the simile of the jigsaw puzzle, it has placed personal values on the sets of pieces and the single pieces. Part of the charm of the game is that other players may set their own values but arrive at the same answer, which, as already said, must be the physician's decision on the individual patient's pathogenesis at the moment of diagnosis. 
We can see how every game from the first has a definite effect on the playing of its successors. We learn gradually to attach special meanings to our own set of pieces which we call physical signs in the light of increasing use of the radiological set; we read new values into our films by applying to them these readjusted physical signs; and we add to both a freshened interest in living pathology. In other words, each game is a new experience, instructive, exhilarating, and whetting to the appetite for further and new problems. The number of these is so large that we need never fear that in our short time we shall solve them all; we have games for a lifetime.

\section{TREATMENT OF HALLUX VALGUS DEFORMITY IN SOLDIERS}

\author{
BY

\section{R. BROOKE, O.B.E., M.S., F.R.C.S.} \\ Lieutenant-Colonel, R.A.M.C.
}

\begin{abstract}
A surgeon who has treated with success hallux valgus deformity of the great toe in civilian patients may be disappointed with the results he obtains when similar methods are used in the case of the soldier. Why should there be this difference? There are many possible explanations. One is that usually in civilian life no question of compensation arises in this type of case, and the patient is thus forced by financial necessity to get back to his job as quickly as possible ; he will co-operate to the fullest extent in the aftertreatment and will make the best of any disability which may remain. In the case of the serving soldier this incentive to get well quickly is absent. He has no financial worries, is usually comfortably housed in an E.M.S. or military hospital, is excused duties, drill, and marching, and yet is not confined to bed and is allowed considerable freedom which he is fit to enjoy. It is not surprising that he takes his time in getting well. $\mathrm{He}$ is unenthusiastic, perhaps lazy, and often perhaps completely uninterested in the treatment. This lack of co-operation may lead to a poor result, which soon becomes apparent to the patient, and on occasion he will exploit it to the full. The greatest caution is therefore called for on the part of the surgeon, especially where operative treatment is contemplated. Each case must be considered on its own merits, and those only selected in whom some degree of co-operation may reasonably be expected, after weeding out men who have a grouse against everything-Army life in particular.
\end{abstract}

An analysis of Army medical categories will show that a man may be placed in Category A1 if he has no foot defects, A2 if he is an A1 man with slight foot defects, $\mathrm{C}$ if he would be fit for a higher category but for marked foot defects. The ultimate aim of any form of treatment is threefold: to raise the soldier to a category higher than that in which he is at present ; to prevent his being placed in a lower category ; and, finally, to prevent his being put in Category $\mathrm{E}$ and boarded out of the Army. Even if his ultimate category is not one of the higher ones, treatment will be worth while provided the time taken over it is not too great.

To be eligible for the lowest category, $\mathrm{C}$, he must be capable of carrying out a full day's work in his own arm for continuous periods without going sick. He must be able to wear and march in Army boots. This does not imply that Army boots are an additional burden which he must bear. They may be heavy but they are well designed, and it is not the boots that are in any way at fault. The soldier, it is true, refers all his foot troubles to his Army boots. He may be boarded Category $E$ because he cannot march in them, but this is only tantamount to saying that no treatment will offer any prospect of his walking in any other than special boots or shoes with reasonable comfort, and probably not even in these. It may perhaps be that through an error of judgment or an accident the treatment attempted has failed in its object, or was possibly unsound in its conception and under war conditions should never have been attempted.

A man working in a sedentary occupation in civil life may for many years carry on in comfort by wearing shoes, only to find that his feet will not stand up to the strain of wearing Army boots. The Army, for self-evident reasons, does not make an issue of shoes. Men who by wearing shoes could be employed in clerical or administrative duties are thus lost to the Service. Special boots, and even such appliances as arch supports, may be obtained in isolated cases, but the formalities connected with their issue are so great that, in practice, attention is confined to an attempt, by palliative measures such as foot drill and massage, and in selected cases operative treatment, to make the patients well enough to wear the ordinary boots supplied without these additions.

\section{Palliative Treatment}

In soldiers, in most cases, the deformity is of the acquired type and is moderate in degree, so that much can be done by well-planned and graduated active exercises, with preliminary thermotherapy, and faradism to the invertors of the foot and intrinsic muscles, which are also at fault. Active exercises may be taught to music, and it is an advantage to group patients together into classes for this purpose. Newcomers are encouraged by the progress which they see in others. A course of daily active foot-exercise classes will last for twenty-one days, and the less severe cases may have recovered sufficiently at the end of that time to return to their units. These are, however, long-term cases, and more than one course may be necessary to tide the patient over the period of foot-strain due to the sudden change from a sedentary occupation to an active Army life.

\section{Operative Treatment}

The patient is a young man and, speaking generally, the deformity is not in its advanced stages and arthritic changes have not yet supervened. There is a well-marked bunion, an outwardly overriding or underriding great toe, a varying degree of stiffness and pain, and an associated dropping of the anterior arch. The deformity is fixed, and cannot be corrected by passive movement. Operative removal of the bunion will ease the pressure of the boot on the tender and sometimes inflamed soft tissues and, followed by a short course of physiotherapy, may be all that is required to enable the man to return to duty and to wear Army boots with comfort.

Such an operation does not correct the valgus deformity of the great toe ; nor, for that matter, do arthroplasties, in which the metatarsal head is partly removed and reshaped or the base of the proximal phalanx removed. Only in older patients with arthritic and painful joints are these operations of Mayo and Brande justifiable-the exception rather than the rule in soldiers - and the most that can be hoped for is that by removing enough bone the joint will be rendered flail and painless. The great toe ceases to act as a lever and is carried as a useless appendage. Failure to remove enough bone gives a painful and stiff jointrather worse, if anything, than it was before. In both these instances the toe will be shortened but the valgus deformity will persist. It is difficult to understand why so much is expected from an arthroplasty of this important weightbearing joint when the results of arthroplasties in other 